\title{
Adaptively Iterative Multiscale Switching Simulation Strategy and Applications to Protein Folding and Structure Prediction
}

Qinglu Zhong ${ }^{1,2}$ and Guohui Li ${ }^{1 *}$

${ }^{1}$ Laboratory of Molecular Modeling and Design, State Key Laboratory of Molecular Reaction Dynamics, Dalian Institute of Chemical Physics, Chinese Academy of Sciences, Dalian 116023, China

${ }^{2}$ University of Chinese Academy of Sciences, Beijing 100049, China

*Correspondence: ghli@dicp.ac.cn 


\section{Contents}

- ART coarse-grained model

$>$ Introduction

> Improvements on hydrogen bond interactions

$>$ Improvements on non-specific hydrophobic interactions

- Simulation details

$>$ Structures

> Adaptive all-atom simulations

$>$ All-atom replica-exchange simulations

$>$ ART coarse-grained replica-exchange simulations

- Analysis methods

$>$ Secondary structure cluster analysis

$>$ Conformational cluster analysis

$>$ Secondary structure combination rules

$>$ TM-score

$>$ Q-score

- Supplementary Figures

- Supplementary References 


\section{ART coarse-grained model}

\section{Introduction}

As proposed in the previous paper ${ }^{1}, A R T$ is a universal user-customized coarse-grained (CG) strategy that can generate a system-specific CG force field anytime and be applied to any system with an arbitrary $C G$ resolution according to research requirements. ART CG model is a two-bead-type model that comprises bonded CG beads (BBs) and nonbonded CG beads (NBs). BBs have only bonded parameters and will be positioned near the molecular backbone to maintain the stability of molecular internal degrees of freedom. NBs will be mainly positioned on side chains or the molecular surface to accurately describe the nonbonded interactions between molecules. Each BB bonds to its neighbor BB. Each NB bonds only to its closest BB to describe the relative motion of the side chain to the backbone. Mapping function and nonbonded parameters can be determined by numerical optimization methods; bonded parameters, including bonds, angles, dihedrals and other constraints, can be determined by Boltzmann Inversion? According to the previous study ${ }^{1}, 1 \mathrm{BB}$ and $1 \mathrm{NB}$ per 1 residue is the optimal resolution for proteins. So this resolution is continued to use in this paper.

\section{Improvements on hydrogen bond interactions}

The previous paper mainly studied inter-molecular recognition, and did not care about intra-molecular conformational changes. Intra-molecular hydrogen bonds were described by bonded Morse potential to keep secondary and tertiary structure stable in ART model. Angles and Dihedral derived from these hydrogen bonds were also involved in bonded terms. However, this paper mainly studies protein folding, which must involve hydrogen bond formation and breaking. Hydrogen bonds are described by nonbonded pairwise Lennard-Jones potential instead this time.

The detailed process of determining parameters is as follows. The optimal CG resolution for protein is one BB and one NB per residue. As described above, BB is near the backbone, NB is on the sidechain. In the bottom-up mapping, all-atom (AA) backbone atoms are corresponding to the $\mathrm{CG} B \mathrm{BB}$ of the same residue, $\mathrm{AA}$ sidechain atoms are corresponding to the CG NB of the same residue. MDTraj ${ }^{3}$ can figure out all hydrogen bonds that occur in AA trajectories and its probability $P$. For each hydrogen bond whose probability $P$ is more than a threshold $P_{\text {min, }}$ a Lennard-Jones potential is added between a pair of CG beads that donor and acceptor correspond to. Donor and acceptor that are within three neighbor residues are excluded. The pairwise distance with maximum probability distribution is set to parameter $\sigma$, the distance corresponding to the minimum of LennardJones potential. On the basis of Boltzmann distribution $p \propto e^{-E / k T}$, the absolute value of the minimum of Lennard-Jones potential is defined as $\varepsilon=c_{1} k T \log \frac{c_{2} P}{P_{\min }}$, where $c_{1}, c_{2}$ are positive coefficients that regulate the strength of interaction. The higher the probability, the stronger the interaction. This can also be done for other alternative interactions as needed, such as $\pi$-interactions, specific hydrophobic interactions. 


\section{Improvements on non-specific hydrophobic interactions}

ART CG nonbonded parameters are optimized by probes in vacuum, so that direct CG nonbonded interactions correspond to AA nonbonded interactions in vacuum. ART CG bonded parameters are optimized from AA trajectories with explicit solvent, so that $C G$ bonded interactions involve solvent effects, but it is only responsible for conformations around the AA trajectories, i.e. the training set. As a consequence, CG does not involve dynamic solvent effects.

As we know, solvent has electrostatic screening effect. If two charged beads are separated by solvent, the interaction between them should be scaled by a relative dielectric constant to imitate those in explicit solvent. Similarly for vdW interactions, if solvent disappears between two separated beads, the two beads lose their vdW interactions with the solvent and must attract each other. Losing screening effect is more like a solvent exclusion process. That is to say, non-screening model is more applicable for hydrophobic interactions, while screening model is more applicable for hydrophilic interactions.

In this paper, therefore, we scale vdW parameter $\varepsilon$ of $C G$ nonbonded bead of hydrophobic residues, ILE, VAL, LEU, PHE, CYS, MET, ALA, TRP, PRO, by 1, i.e. vacuum vdW potential; and scale $\varepsilon$ of $C G$ nonbonded bead of the other residues by 0.125 , i.e. screening $v d W$ potential. It is more effective for CG folding dominated by hydrophobic interactions.

\section{Simulation details}

\section{Structures}

All the reference structures involved in this paper are illustrated in Figure 6. The random coiled initial structures are modelled by MODELLER ${ }^{4}$. All the simulations are performed by GROMACS 5 .

\section{Adaptive all-atom simulations}

Each molecule was positioned into a cubic box extended $1 \mathrm{~nm}$ from the solute surface and dissolved with TIP3P_L waters ${ }^{6}$, which are the TIP3P model with a mass of 3 a.m.u. for the oxygen atom and 1.5 a.m.u. for the hydrogen atoms. $0.15 \mathrm{M}$ chloride and sodium ions were added to neutralize the net charge of the system. All AA simulations were performed with Charmm $36 \mathrm{~m}^{7}$ force field, which has a better effect on protein folding ${ }^{8}$. The system was subjected to energy minimization using the steepest descent algorithm, then gradually heated to the target temperature under NVT ensemble and equilibrated under NPT ensemble. The step size of leapfrog verlet integrator was set to $2.5 \mathrm{fs}$. Particle-Mesh Ewald ${ }^{9}$ method was utilized to treat the electrostatic interactions with cutoff distance of 1.1 $\mathrm{nm}$. The same cutoff value was chosen for treating the van der Waals interactions. LINCS ${ }^{10}$ algorithm was used to constrain covalent bonds involving hydrogen atoms. The NoseHoover ${ }^{11,12}$ Thermostat was applied to couple temperature at the target temperature in the system every 2 ps. The Parrinello-Rahman ${ }^{13}$ Barostat was used to control pressure in the 
system at 1 bar. Each production run was performed in the adaptive mode. The target temperatures are $300 \mathrm{~K}$ for Trp-cage, $310 \mathrm{~K}$ for $\mathrm{BBL}, 330 \mathrm{~K}$ for the others. The simulation times are listed in Table 1.

\section{All-atom replica-exchange simulations}

The optional AA replica-exchange simulations at Step 11 were performed with 12 replicas at $303.00 \mathrm{~K}, 308.62 \mathrm{~K}, 314.35 \mathrm{~K}, 320.18 \mathrm{~K}, 326.12 \mathrm{~K}, 332.17 \mathrm{~K}, 338.33 \mathrm{~K}, 344.60 \mathrm{~K}, 351.00 \mathrm{~K}$, $357.51 \mathrm{~K}, 364.14 \mathrm{~K}, 370.90 \mathrm{~K}$, respectively. Replica exchange was attempted every $2.5 \mathrm{ps}$. Exchange probability was about $5 \sim 10 \%$. Other MD options were the same as the above AA simulations. According to the description of Step 11, more than one representative conformation would get involved. If 2 conformations $A B$ get involved, there are 2 permutations to put 2 conformations into 12 replicas in turn, $A B A B A B A B A B A B$, $B A B A B A B A B A B A$. If 3 conformations $A B C$ get involved, there are 6 permutations, ABCABCABCABC, BCABCABCABCA, CABCABCABCAB, ACBACBACBACB, CBACBACBACBA, BACBACBACBAC. And so on. The aim is to eliminate the effect of different conformations at different initial temperatures.

\section{ART coarse-grained replica-exchange simulations}

The initial CG conformation could be any random structure, which had almost nothing impact on results. All CG replica-exchange simulations were performed with 10 replicas at $300 \mathrm{~K}, 340 \mathrm{~K}, 390 \mathrm{~K}, 450 \mathrm{~K}, 520 \mathrm{~K}, 600 \mathrm{~K}, 690 \mathrm{~K}, 790 \mathrm{~K}, 900 \mathrm{~K}, 1020 \mathrm{~K}$, respectively. The step size of Langevin integrator was set to $10 \mathrm{fs}$. The temperature was controlled using $\mathrm{V}$ rescale ${ }^{14}$ thermostat with a coupling constant of $10 \mathrm{fs}$. The relative dielectric constant of Coulomb interaction with reaction-field ${ }^{15}$ was modified from 16 to 80 gradually within 2.4 $\mathrm{nm}$, and fixed 80 beyond $2.4 \mathrm{~nm}$. The cutoff of van der Waals interactions was set to 2.4 $\mathrm{nm}$. Replica exchange was attempted every 100 ps. Exchange probability was about $10 \%$ $\sim 30 \%$.

\section{Analysis methods}

\section{Secondary structure cluster analysis}

For each frame in the AA trajectories, a sequence of secondary structure type for each residue can be analyzed by DSSP method. We denote helix as 1 , strand as -1 , coil as 0 . The secondary structure of each frame can be represented by a residue-numberdimensional ternary vector. All of the nonzero vectors will be performed cluster analysis using DBSCAN ${ }^{16}$ algorithm with Bray-Curtis ${ }^{17}$ metric. DBSCAN algorithm is adept in finding core samples of high density. The Bray-Curtis distance is effective for measuring the dissimilarity between two species. As a result, conformations with high-density similar secondary structures become a cluster. 


\section{Conformational cluster analysis}

Conformations are performed cluster analysis using Gromos algorithm with RMSD metric. This algorithm is one of the alternatives in GROMACS. See details in GROMACS manual (http://manual.gromacs.org/archive/5.0.7/programs/gmx-cluster.html) or its reference ${ }^{18}$.

\section{Secondary structure combination rules}

The secondary structure of one conformation is represented by a ternary vector. The secondary structure of a cluster of conformations should be the average of all the vector. The cluster-vector value of each element is between -1 and 1 . The element tends to 1 means the residue tends to helix; the element tends to -1 means the residue tends to strand.

We define COMPATIBLE as that the absolute value of the difference between two clustervectors is not more than 1 . It means that one residue should not tend to both helix and strand, otherwise the two clusters are not COMPATIBLE.

Combination rules. In Step 6, the total $N_{\text {clust }}$ S-clusters can be separated into two sets, set A has $N_{\mathrm{A}}$ clusters, set B has $N_{\mathrm{B}}$ clusters, $N_{\text {clust }}=N_{\mathrm{A}}+N_{\mathrm{B}}$. If any two elements of set $\mathrm{A}$ are COMPATIBLE, and any element of set $B$ is not COMPATIBLE with at least one element of set $A$, then all the elements of set $A$ can be combined into a group, i.e. G-CGFF.

Force field combination algorithm. The combinational G-CGFF is defined as the weighted average of all S-CGFFs to be combined. G-CGFF $=\sum_{i} w_{i}$ S-CGFF ${ }_{i}$, where $w_{i}$ is the weighted coefficient. In practice, the force constant of each potential term can be scaled by $w_{i}$ and each term of each S-CGFF $i$ can be appended to G-CGFF one by one. In this paper, weighted coefficients are set to be uniform.

\section{TM-score}

$$
\begin{gathered}
T M=\operatorname{Max}\left[\frac{1}{N} \sum_{i}^{N} \frac{1}{1+\left(\frac{d_{i}}{d_{0}}\right)^{2}}\right] \\
d_{0}=1.24 \sqrt[3]{N-15}-1.8
\end{gathered}
$$

Where $N$ is the protein sequence length, $d_{i}$ is the distance between the $i$-th pair of aligned residues, $d_{0}$ is a scale to normalize the match difference. "Max" denotes the maximum value after optimal spatial superposition. TM-score always lies between 0 and 1 . If a structure is more similar to the reference structure, TM-score is closer to 1, otherwise closer to 0 . Protein pairs with a TM-score $>0.5$ are mostly in the same fold, while those with a TMscore $<0.5$ are mainly not in the same fold ${ }^{19}$. Due to the definition of $d_{0}$, TM-score is unconvinced whose ordered sequence length is less than 20 , such as the Trp-cage and BBL in this paper. 


\section{Q-score}

$$
Q=\frac{1}{N} \sum_{i}^{N} \frac{1}{1+e^{d_{i}-d_{i}^{0}-0.1}}
$$

For $N$ contacts of residual pairs, $d_{i}$ is the current distance between the Ca atoms of the $i-$ th residual pair, $d_{i}{ }^{0}$ is the same distance in the reference structure (measured in the unit of $\mathrm{nm}$ ). Q-score always lies between 0 and 1 . If a structure is more similar to the reference structure, $Q$-score is closer to 1, otherwise closer to 0 . But $Q$ is insensitive in the phases between secondary and tertiary structures, so that $Q$ is better for low-order structure evaluations. 


\section{Supplementary Figures}

a

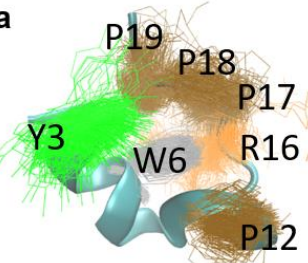

b

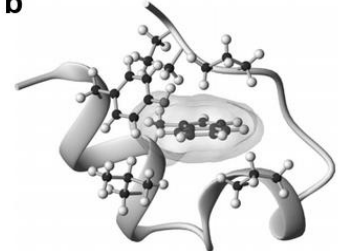

Figure S1. Tertiary structure of Trp-cage. (a) Demonstration of the predicted conformational ensemble. (b) Demonstration of the experimental structure ${ }^{20}$. 

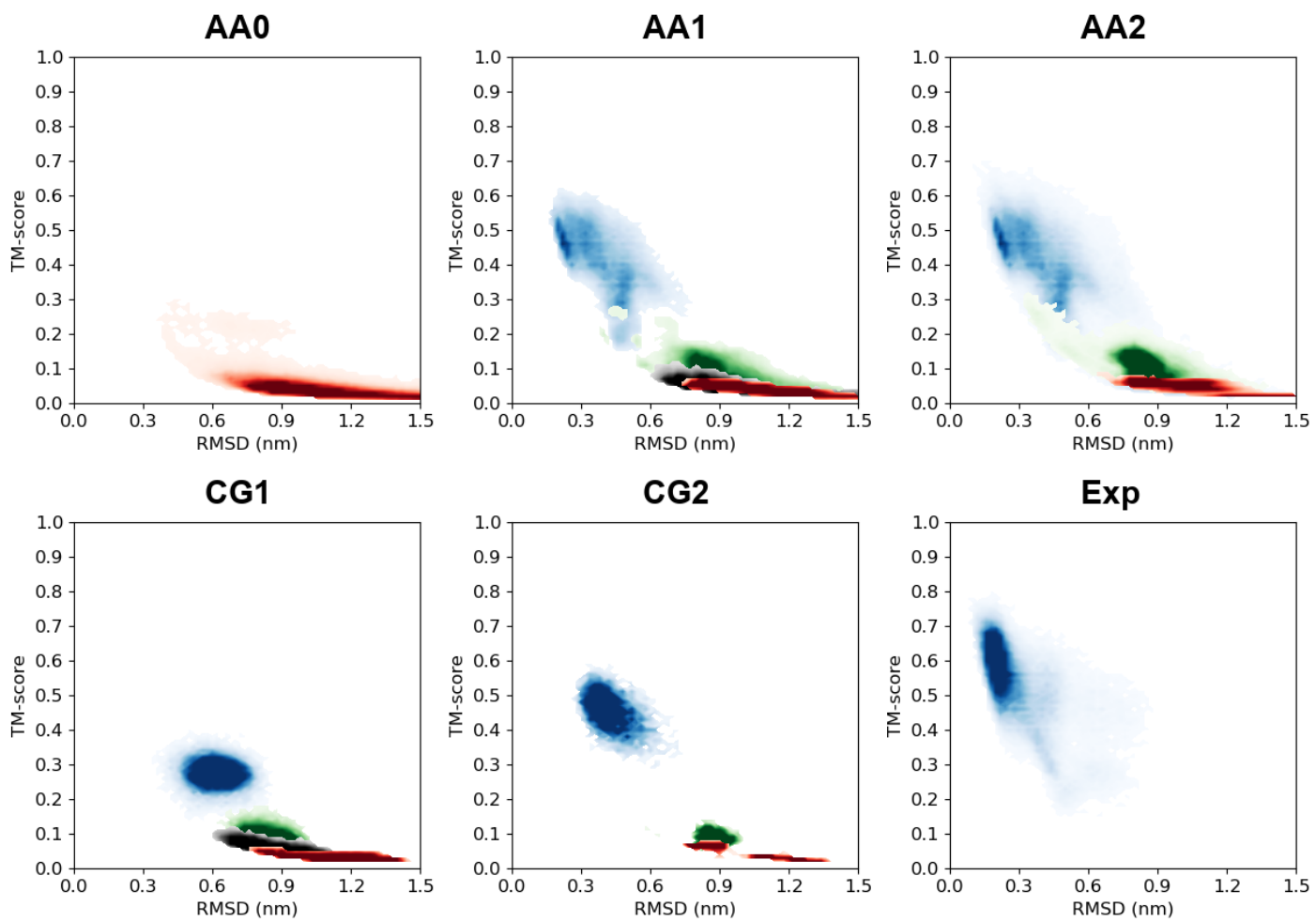

Figure S2. Ab initio prediction process of WW domain compared with the experimental structure. RMSD and TM-score of trajectories are shown in a 2D map for each AIMS step and $A A$ simulation from the experimental structure. Color depth denotes conformational distribution. Colors are corresponding to Figure 4: red for Group ABD, black for Group AE, green for Group CD, blue for Group CE. 

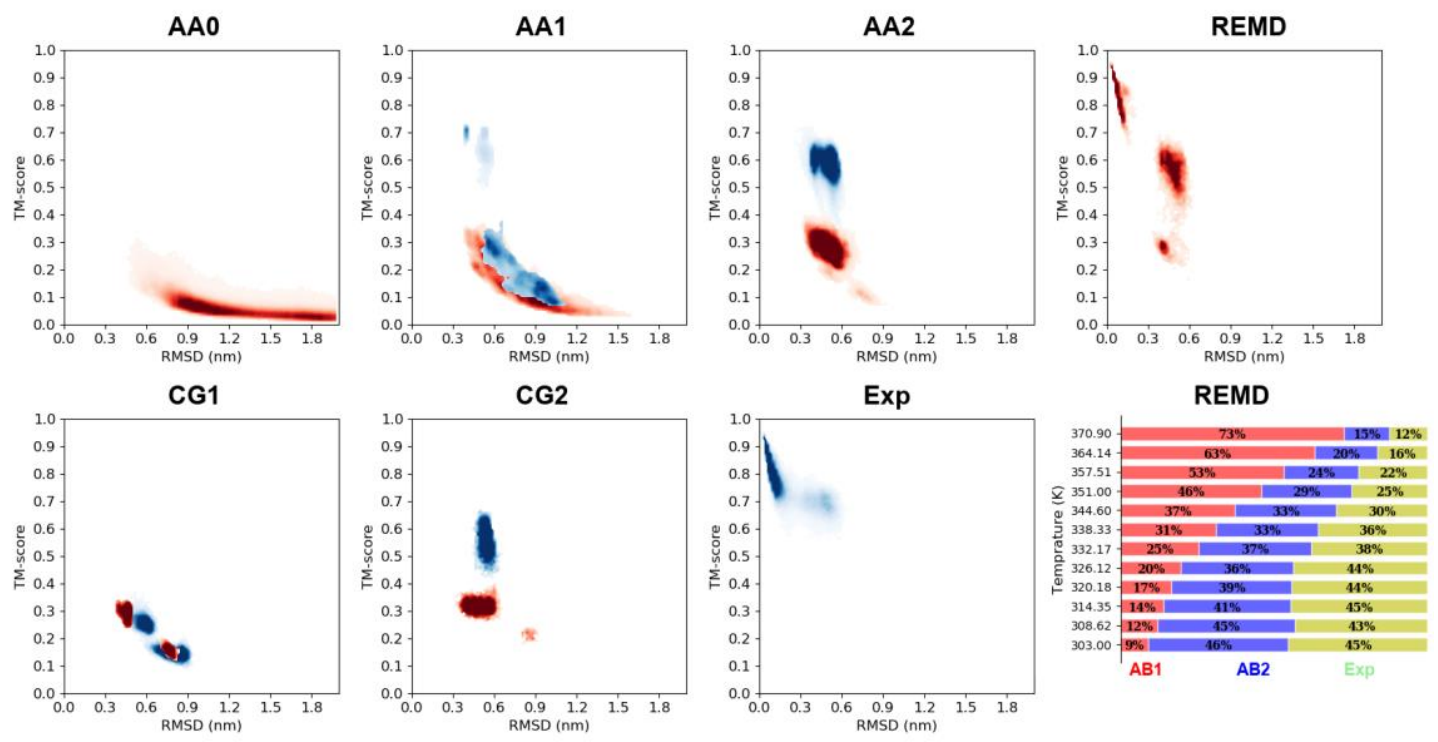

Figure S3. Ab initio prediction process of NTL9 compared with the experimental structure. RMSD and TM-score of trajectories are shown in a 2D map for each AIMS step and AA simulation from the experimental structure. Color depth denotes conformational distribution. Colors are corresponding to Figure 5: red for Group AB1, blue for Group AB2. The rightmost panel shows the result of $A A$ REMD simulations for Group AB1, $A B 2$ and the experimental structure. 
a

은

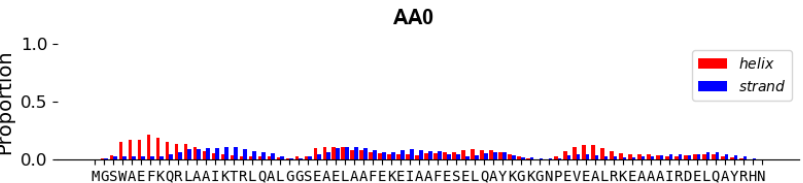

Cluster analysis for AA0

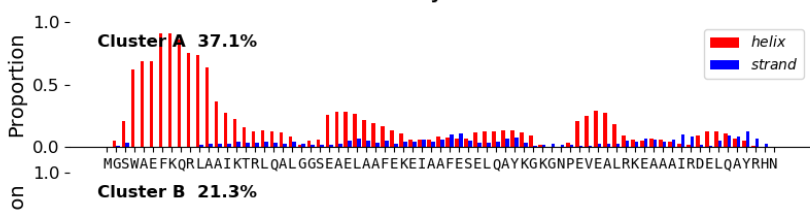

ᄃ

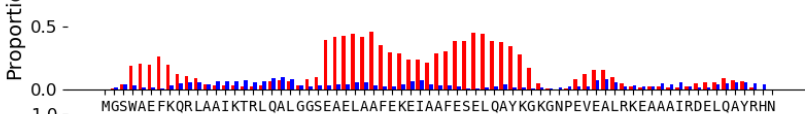

CAAIKTRLOALGGSEAELAAFEKEIAAFESELOAYKGKGNPEVEA

$\overbrace{}^{1.0-}$ Cluster C $\mathbf{1 1 . 5 \%}$

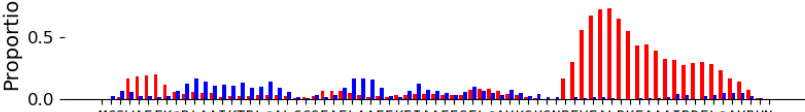

0.0 MGSWAEFKQR LAAIKTRL OAL GGSEAELAAFEKEIAAFESEL QAYKGKGNPEVEALRKEAAAIRDEL QAYRHN

은

Cluster D 9.1ph

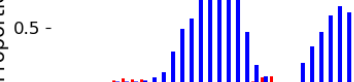

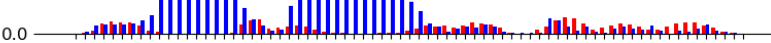

SWAEFKQR LAAIKTRL QAL GGSEAELAAFEKEIAAFESEL QAYKGKGNPEVEALRKEAAAIRDELQAY

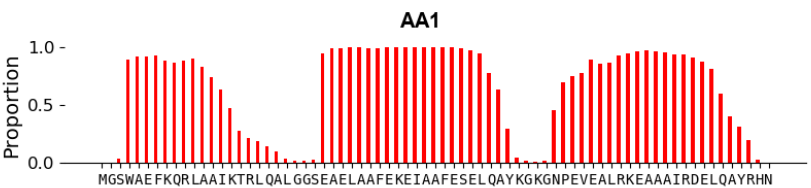

AA2

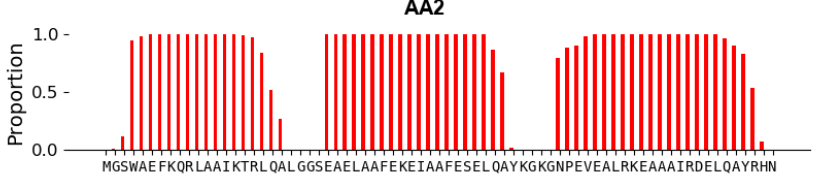

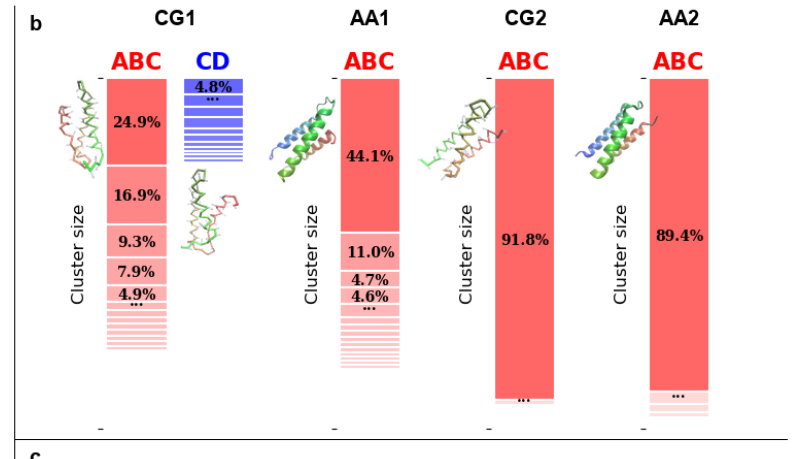

c
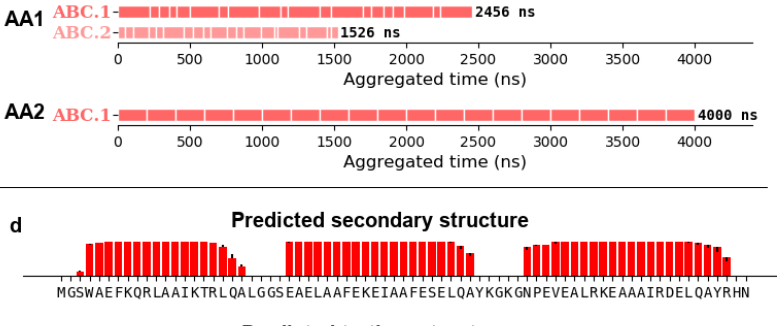

Predicted tertiary structure

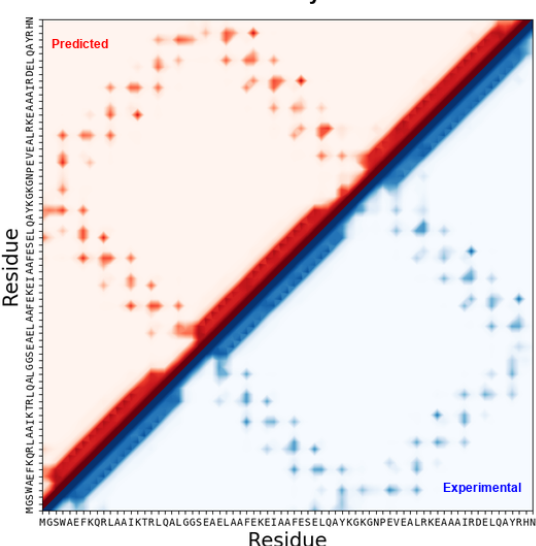

Figure S4. Ab initio prediction process of a3D. (a) Secondary structure distribution of trajectories. (b) Conformational cluster analysis and representative conformations of main clusters. (c) Aggregated times of adaptive AA simulations. (d) Secondary and tertiary structure of the predicted conformational ensemble, and comparisons with those initiated from the experimental structure. Upper, bars for the predicted and black lines over the bars for differences between the predicted and the experimental; lower, ref for the predicted and blue for the experimental. 

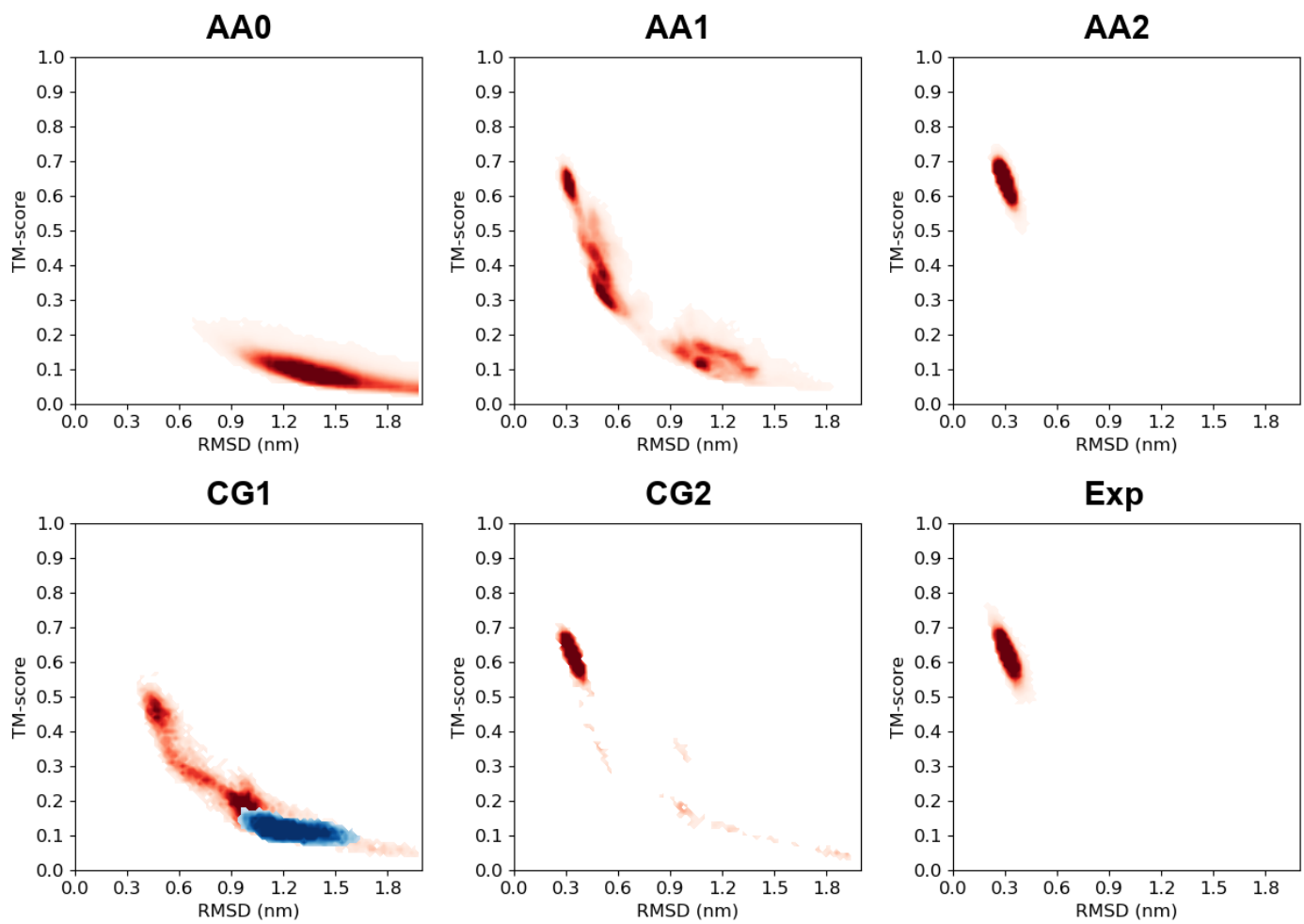

Figure S5. Ab initio prediction process of a3D compared with the experimental structure. RMSD and TM-score of trajectories are shown in a 2D map for each AIMS step and AA simulation from the experimental structure. Color depth denotes conformational distribution. Colors are corresponding to Figure S4: red for Group ABC, blue for Group CD. 
a

은
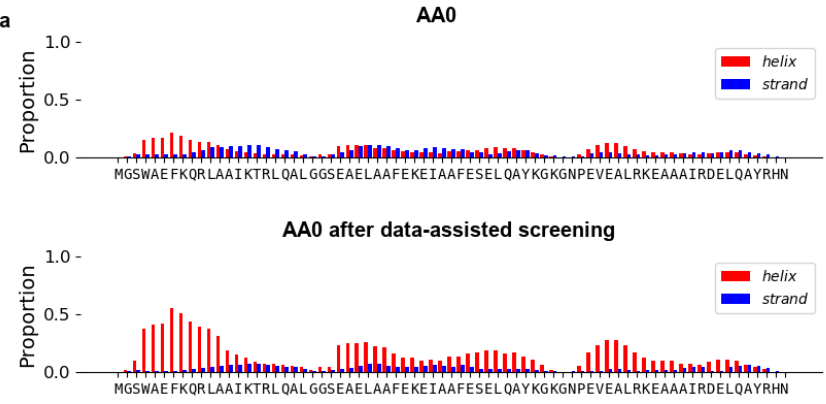

Cluster analysis for AA0

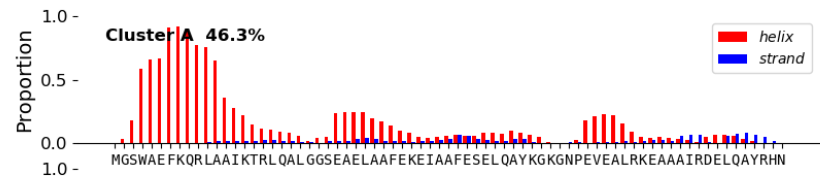

든

Cluster B $\mathbf{2 4 . 4 \%}$

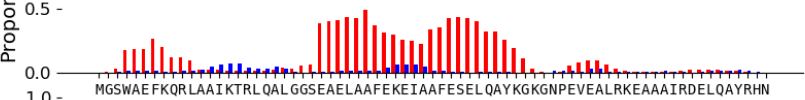
1.0 - MGSWAEFKORLAAIKT
Cluster C $\mathbf{1 1 . 9 \%}$

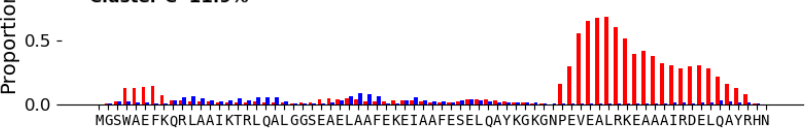

AA1

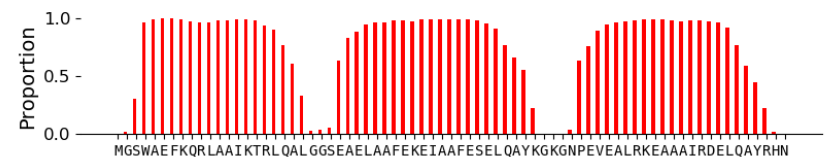

AA2

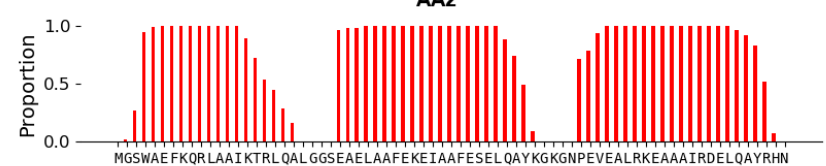

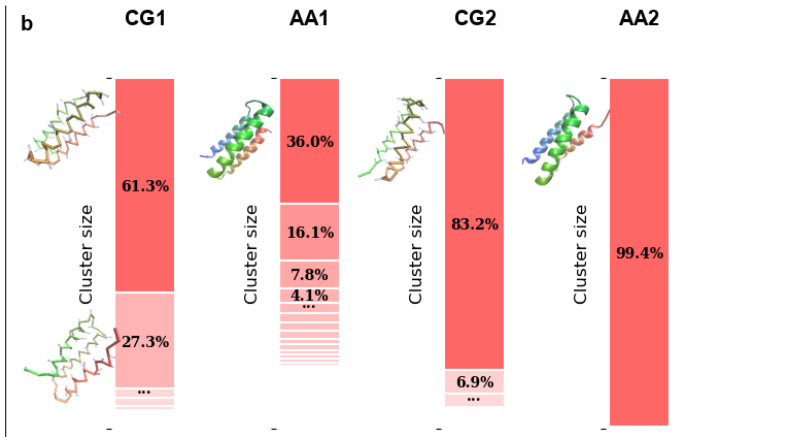
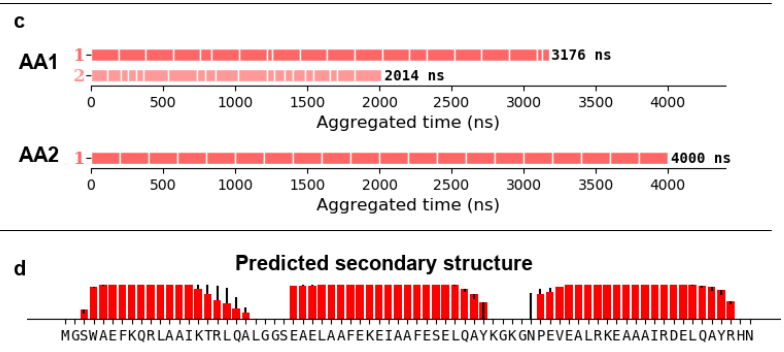

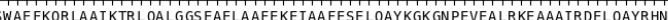

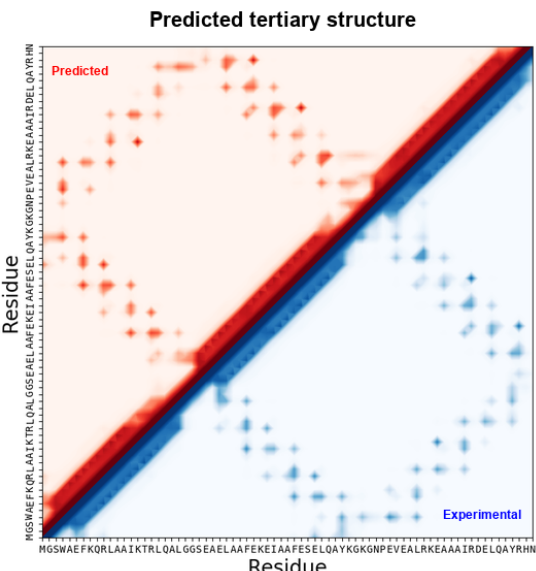

Figure S6. Data-assisted prediction process of a3D. (a) Secondary structure distribution of trajectories. (b) Conformational cluster analysis and representative conformations of main clusters. (c) Aggregated times of adaptive AA simulations. (d) Secondary and tertiary structure of the predicted conformational ensemble, and comparisons with those initiated from the experimental structure. Upper, bars for the predicted and black lines over the bars for differences between the predicted and the experimental; lower, ref for the predicted and blue for the experimental. 

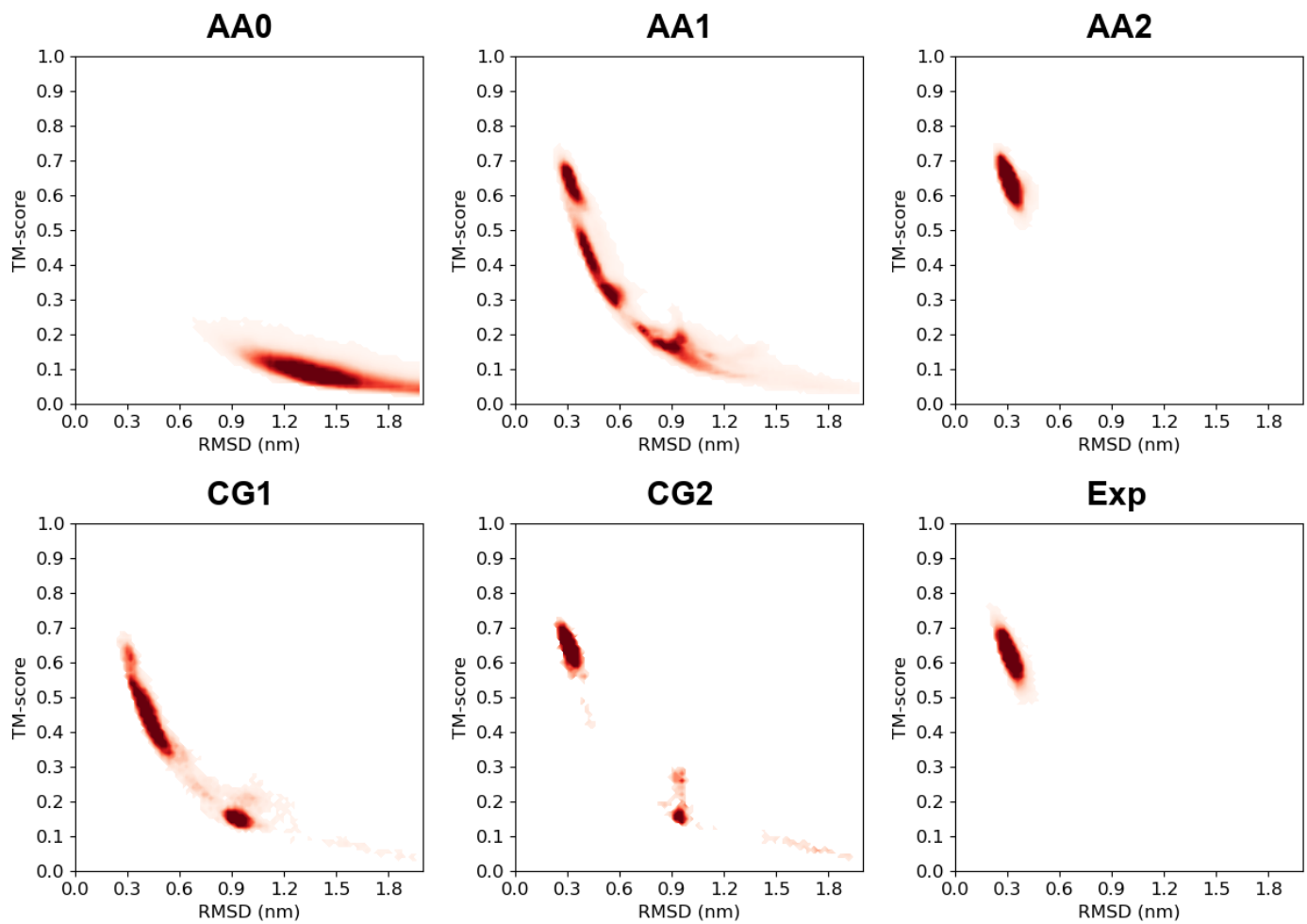

Figure S7. Data-assisted prediction process of $\alpha 3 \mathrm{D}$ compared with the experimental structure. RMSD and TM-score of trajectories are shown in a 2D map for each AIMS step and $A A$ simulation from the experimental structure. Color depth denotes conformational distribution. 
a

을
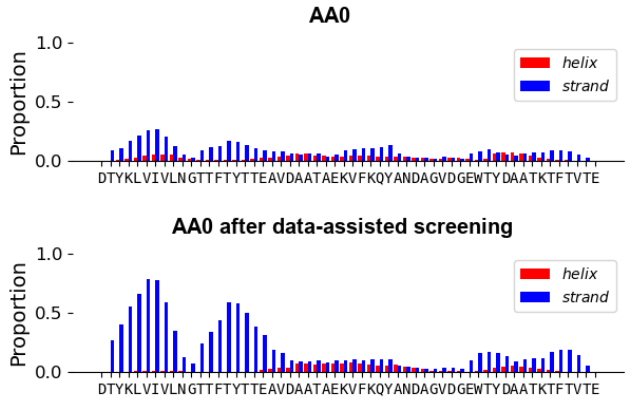

Cluster analysis for AA0

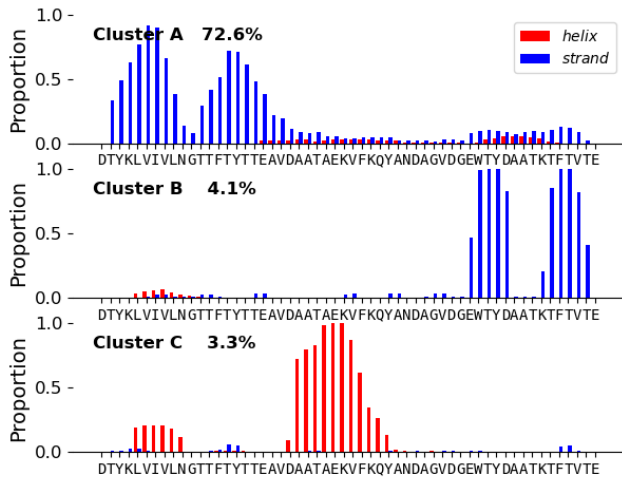

AA1

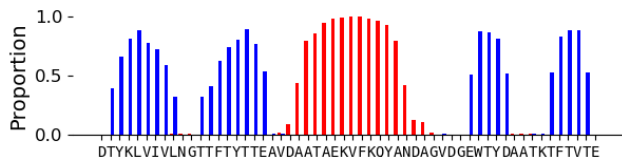

AA2

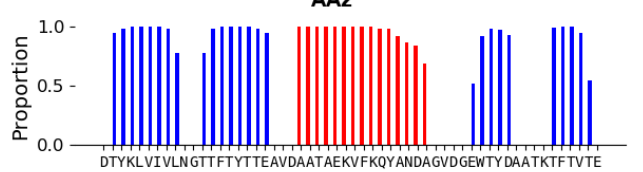

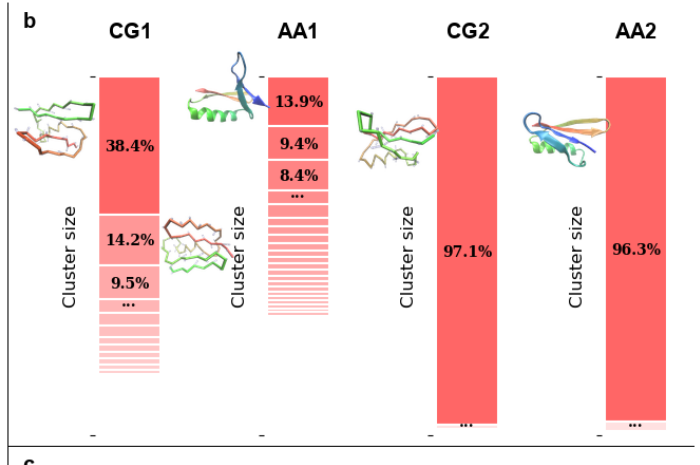

c

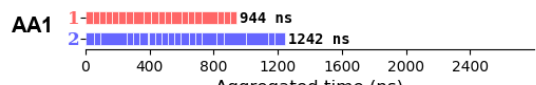

AA2

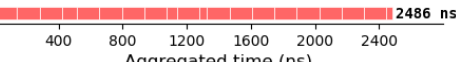
time (ns)

d Predicted secondary structure

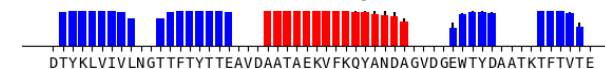

Predicted tertiary structure

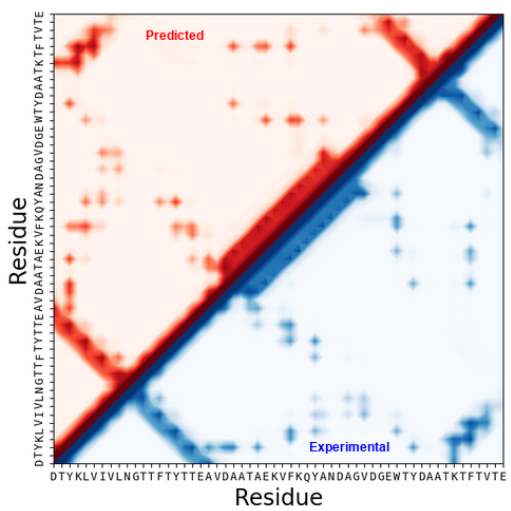

Figure S8. Data-assisted prediction process of Protein G. (a) Secondary structure distribution of trajectories. (b) Conformational cluster analysis and representative conformations of main clusters. (c) Aggregated times of adaptive AA simulations. (d) Secondary and tertiary structure of the predicted conformational ensemble, and comparisons with those initiated from the experimental structure. Upper, bars for the predicted and black lines over the bars for differences between the predicted and the experimental; lower, ref for the predicted and blue for the experimental. 

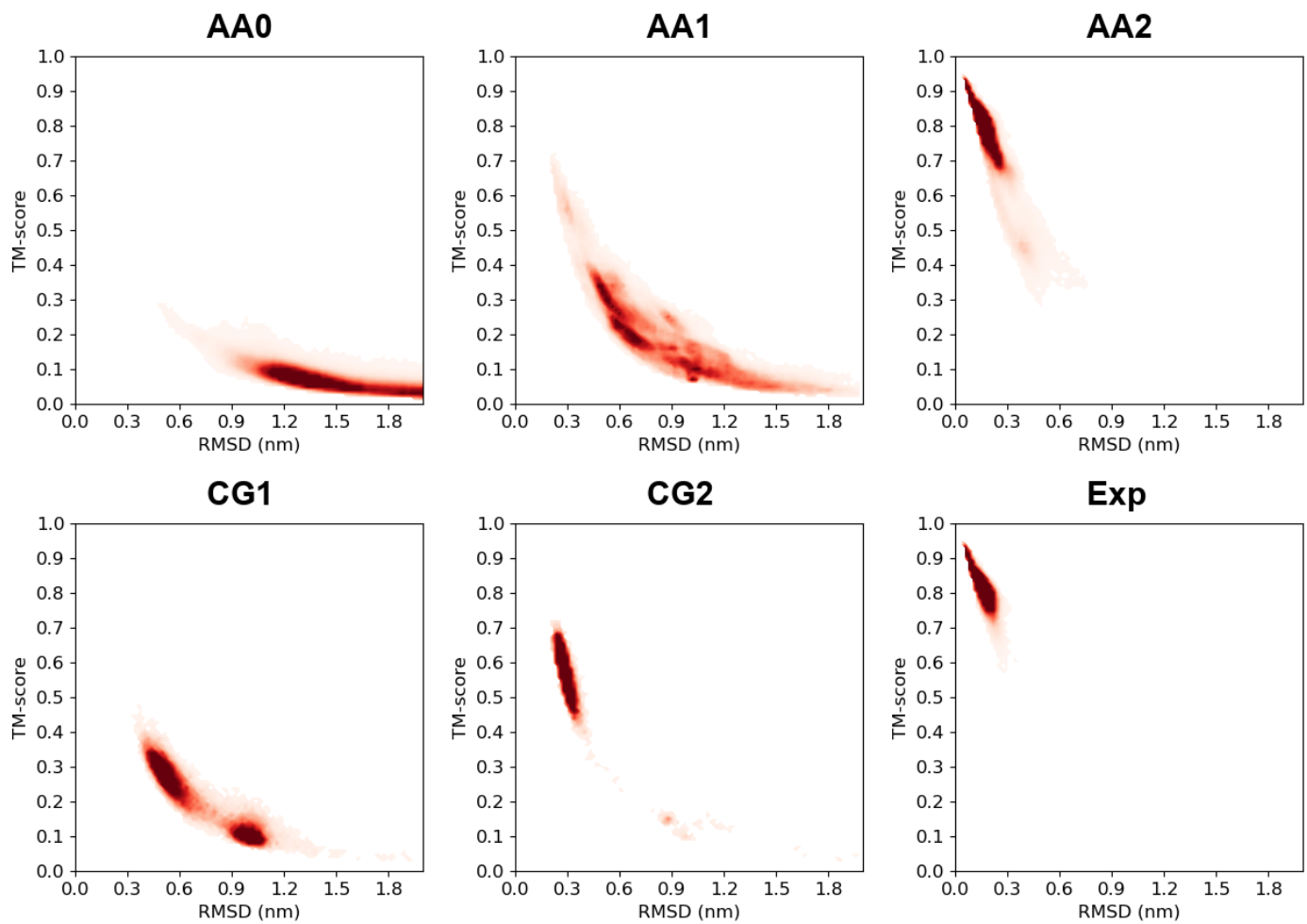

Figure S9. Data-assisted prediction process of Protein $\mathrm{G}$ compared with the experimental structure. RMSD and TM-score of trajectories are shown in a 2D map for each AIMS step and $A A$ simulation from the experimental structure. Color depth denotes conformational distribution. 


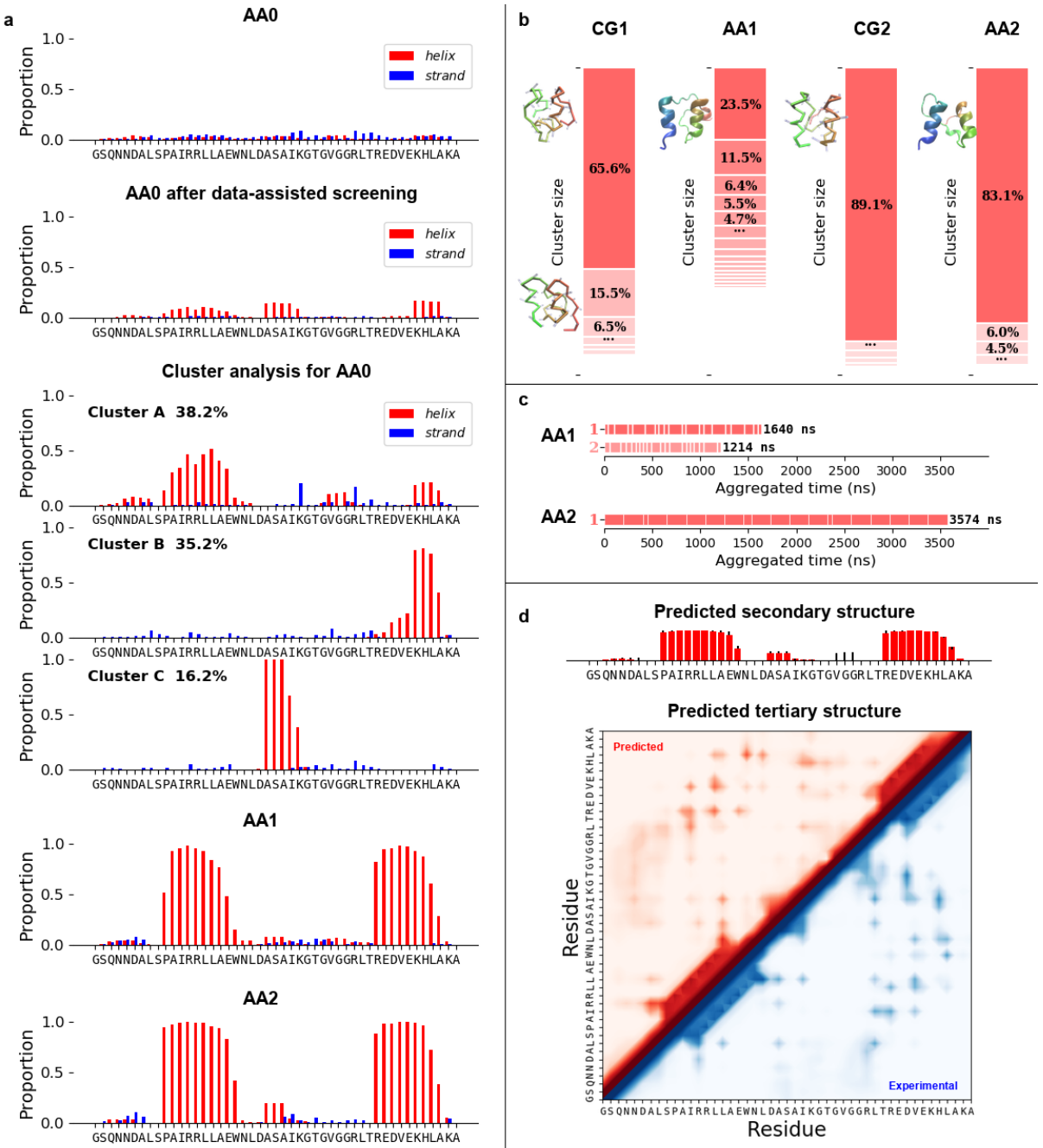

Figure S10. Data-assisted prediction process of BBL. (a) Secondary structure distribution of trajectories. (b) Conformational cluster analysis and representative conformations of main clusters. (c) Aggregated times of adaptive AA simulations. (d) Secondary and tertiary structure of the predicted conformational ensemble, and comparisons with those initiated from the experimental structure. Upper, bars for the predicted and black lines over the bars for differences between the predicted and the experimental; lower, ref for the predicted and blue for the experimental. 

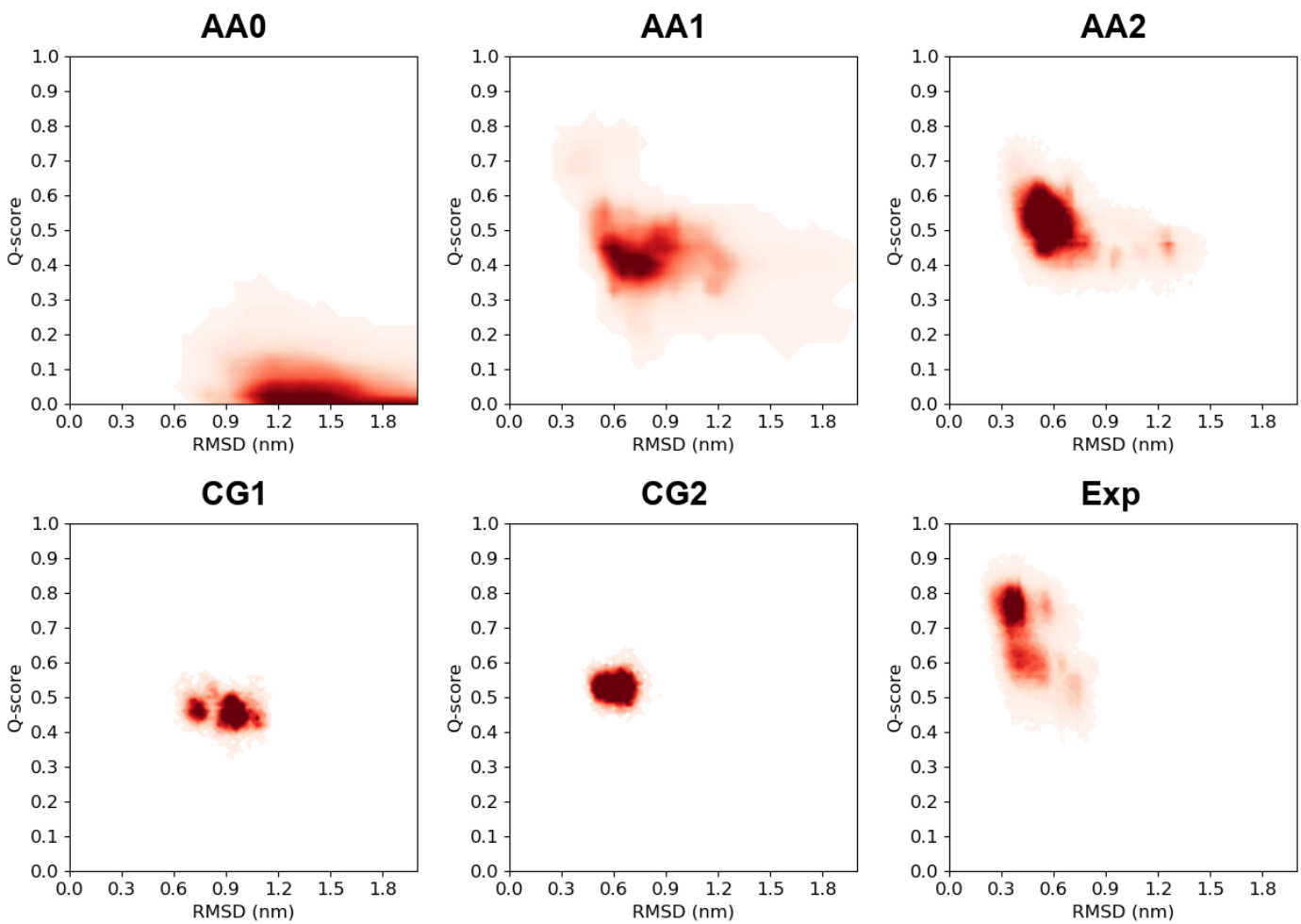

Figure S11. Data-assisted prediction process of BBL compared with the experimental structure. RMSD and Q-score of trajectories are shown in a 2D map for each AIMS step and $A A$ simulation from the experimental structure. Color depth denotes conformational distribution. 


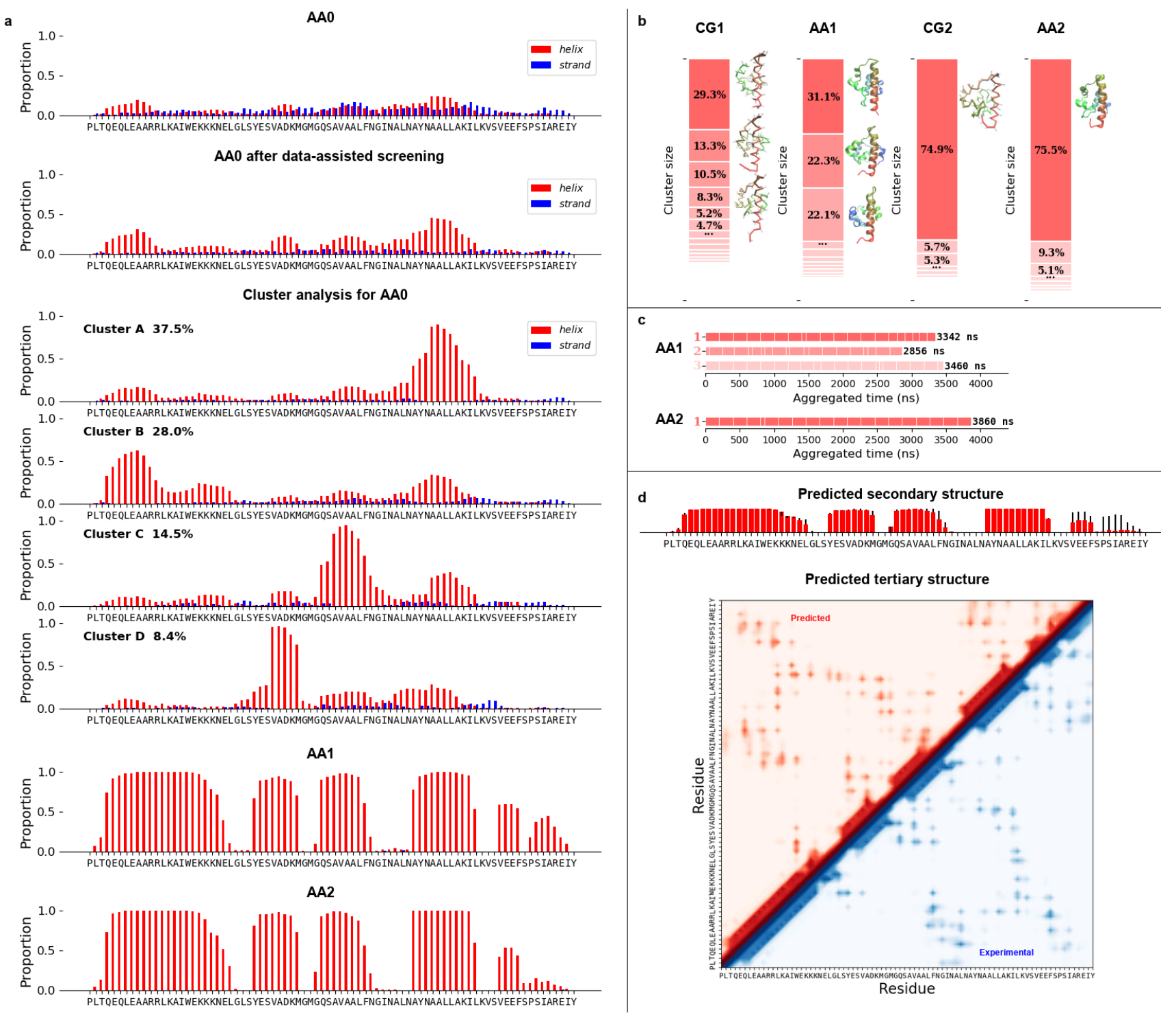

Figure S12. Data-assisted prediction process of $\lambda$-repressor. (a) Secondary structure distribution of trajectories. (b) Conformational cluster analysis and representative conformations of main clusters. (c) Aggregated times of adaptive AA simulations. (d) Secondary and tertiary structure of the predicted conformational ensemble, and comparisons with those initiated from the experimental structure. Upper, bars for the predicted and black lines over the bars for differences between the predicted and the experimental; lower, ref for the predicted and blue for the experimental. 

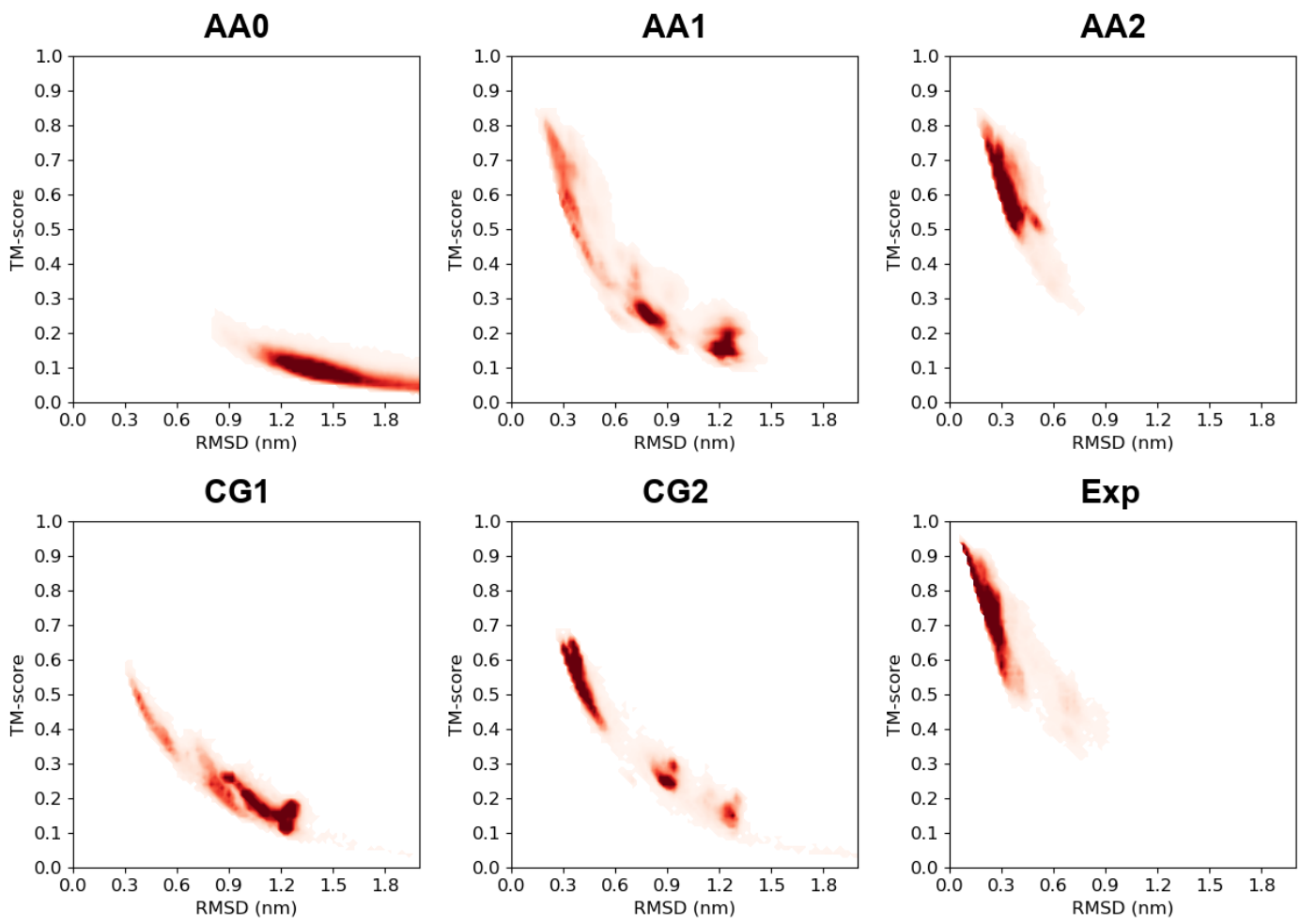

Figure S13. Data-assisted prediction process of $\lambda$-repressor compared with the experimental structure. RMSD and TM-score of trajectories are shown in a 2D map for each AIMS step and AA simulation from the experimental structure. Color depth denotes conformational distribution. 
Sequence MGSWAEFKQRLAAIKTRLQALGGSEAELAAFEKEIAAFESELQAYKGKGNPEVEALRKEAAAIRDELQAYRHN Experi. CHHHHHHHHHHHHHHHHHHHCCCHHHCHHHHHHHHHHHHHHHHHCCCCCCCCCCHHHHHHHHHHHHHHHHCCC PSIPRED CCCHHHHHHHHHHHHHHHHHHCCCHHHHHHHHHHHHHHHHHHHHHCCCCCHHHHHHHHHHHHHHHHHHHHHCC

Protein G Similarity $=91.1 \%$

Sequence DTYKLVIVLNGTTFTYTTEAVDAATAEKVFKQYANDAGVDGEWTYDAATKTFTVTE Experi. CEEEEEEECCCCEEEEEEECCCHHHHHHHHHHHHHCCCCCCEEEEECCCCEEEEEC PSIPRED CEEEEEEEECCCCEEEEEECCCHHHHHHHHHHHHHHHCCCCCCEEECCCCCEEEEEC

BBL

Similarity $=95.7 \%$

Sequence GSQNNDALSPAIRRLLAEWNLDASAIKGTGVGGRLTREDVEKHLAKA

Experi. CCCCCCCCCHHHHHHHHHCCCCHHHCCCCCCCCCCCHHHHHHHHHCC

PSIPRED CCCCCCCCCHHHHHHHHHHCCCHHHCCCCCCCCCCCHHHHHHHHHHC

$\lambda$-repressor Similarity $=97.5 \%$

Sequence PLTQEQLEAARRLKAIWEKKKNELGLSYESVADKMGMGQSAVAALFNGINALNAYNAALLAKILKVSVEEFSPSIAREIY Experi. CCCHHHHHHHHHHHHHHHHHHHHHCCCHHHHHHHHCCCHHHHHHHHCCCCCCCHHHHHHHHHHHCCCHHHCCHHHHHHHC PSIPRED CCCHHHHHHHHHHHHHHHHHHHHCCCCHHHHHHHHCCCHHHHHHHHCCCCCCCHHHHHHHHHHHCCCHHHHCHHHHHHHC

Figure S14. Comparison of secondary structure between the experimental structure and PSIPRED prediction.

a

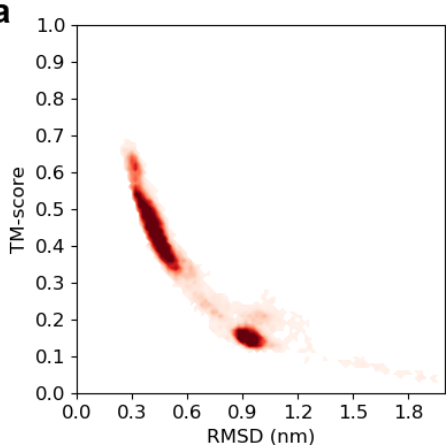

b

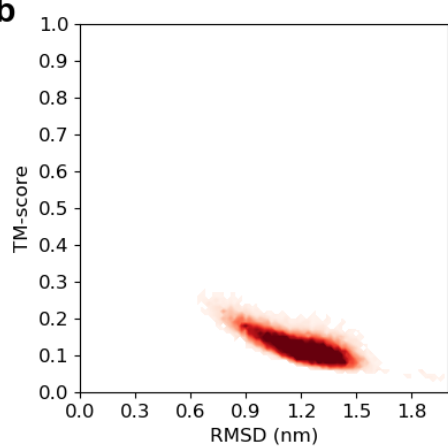

Figure S15. Comparison between (a) with and (b) without secondary structure cluster analysis. RMSD and TM-score of a3D CG1 trajectories are shown in a 2D map. 


\section{Supplementary References}

1 Zhong, Q. \& Li, G. Arbitrary Resolution with Two Bead Types Coarse-Grained Strategy and Applications to Protein Recognition. Journal of Physical Chemistry Letters 11, 3263-3270, doi:10.1021/acs.jpclett.0c00750 (2020).

2 Ruehle, V., Junghans, C., Lukyanov, A., Kremer, K. \& Andrienko, D. Versatile ObjectOriented Toolkit for Coarse-Graining Applications. Journal of Chemical Theory and Computation 5, 3211-3223, doi:10.1021/ct900369w (2009).

3 McGibbon, R. T. et al. MDTraj: A Modern Open Library for the Analysis of Molecular Dynamics Trajectories. Biophysical Journal 109, 1528-1532, doi:10.1016/j.bpj.2015.08.015 (2015).

4 Webb, B. \& Sali, A. Comparative Protein Structure Modeling Using MODELLER. Current protocols in bioinformatics 54, 5.6.1-5.6.37, doi:10.1002/cpbi.3 (2016).

5 Van der Spoel, D. et al. GROMACS: Fast, flexible, and free. Journal of Computational Chemistry 26, 1701-1718, doi:10.1002/jcc.20291 (2005).

6 Mancini, M. et al. Observation of chiral edge states with neutral fermions in synthetic Hall ribbons. Science 349, 1510-1513, doi:10.1126/science.aaa8736 (2015).

7 Huang, J. et al. CHARMM36m: an improved force field for folded and intrinsically disordered proteins. Nature Methods 14, 71-73, doi:10.1038/nmeth.4067 (2017).

8 Wang, A., Zhang, Z. \& Li, G. Higher Accuracy Achieved in the Simulations of Protein Structure Refinement, Protein Folding, and Intrinsically Disordered Proteins Using Polarizable Force Fields. Journal of Physical Chemistry Letters 9, 7110-7116, doi:10.1021/acs.jpclett.8b03471 (2018).

9 Essmann, U. et al. A SMOOTH PARTICLE MESH EWALD METHOD. Journal of Chemical Physics 103, 8577-8593, doi:10.1063/1.470117 (1995).

10 Hess, B., Bekker, H., Berendsen, H. J. C. \& Fraaije, J. LINCS: A linear constraint solver for molecular simulations. Journal of Computational Chemistry 18, 1463-1472, doi:10.1002/(sici)1096-987x(199709)18:12<1463::aid-jcc4>3.0.co;2-h (1997).

Nose, S. A MOLECULAR-DYNAMICS METHOD FOR SIMULATIONS IN THE CANONICAL ENSEMBLE. Molecular Physics 52, 255-268, doi:10.1080/00268978400101201 (1984).

12 Hoover, W. G. CANONICAL DYNAMICS - EQUILIBRIUM PHASE-SPACE DISTRIBUTIONS. Physical Review A 31, 1695-1697, doi:10.1103/PhysRevA.31.1695 (1985).

13 Parrinello, M. \& Rahman, A. POLYMORPHIC TRANSITIONS IN SINGLE-CRYSTALS - A NEW MOLECULAR-DYNAMICS METHOD. Journal of Applied Physics 52, 71827190, doi:10.1063/1.328693 (1981).

14 Bussi, G., Donadio, D. \& Parrinello, M. Canonical sampling through velocity rescaling. Journal of Chemical Physics 126, doi:10.1063/1.2408420 (2007).

15 Tironi, I. G., Sperb, R., Smith, P. E. \& Vangunsteren, W. F. A GENERALIZED REACTION FIELD METHOD FOR MOLECULAR-DYNAMICS SIMULATIONS. Journal of Chemical Physics 102, 5451-5459, doi:10.1063/1.469273 (1995).

16 Schubert, E., Sander, J., Ester, M., Kriegel, H.-P. \& Xu, X. DBSCAN Revisited, Revisited: Why and How You Should (Still) Use DBSCAN. Acm Transactions on Database Systems 42, doi:10.1145/3068335 (2017). 
17 Bray, J. R. \& Curtis, J. T. AN ORDINATION OF THE UPLAND FOREST COMMUNITIES OF SOUTHERN WISCONSIN. Ecological Monographs 27, 326-349 (1957).

18 Daura, X. et al. Peptide folding: When simulation meets experiment. Angewandte Chemie-International Edition 38, 236-240, doi:10.1002/(sici)15213773(19990115)38:1/2<236::aid-anie236>3.0.co;2-m (1999).

$19 \mathrm{Xu}, \mathrm{J}$. \& Zhang, Y. How significant is a protein structure similarity with TM-score $=0.5$ ? Bioinformatics 26, 889-895, doi:10.1093/bioinformatics/btq066 (2010).

20 Barua, B. et al. The Trp-cage: optimizing the stability of a globular miniprotein. Protein Engineering Design \& Selection 21, 171-185, doi:10.1093/protein/gzm082 (2008). 